\title{
EDITORIAL
}

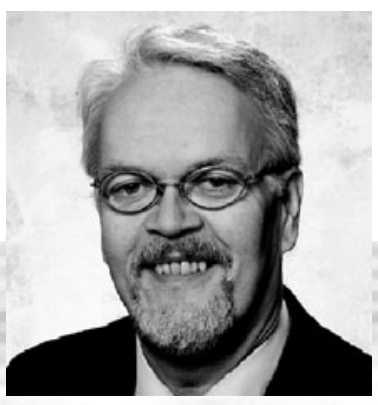

Hermann Engesser

Chefredakteur

\section{Nervensystem unserer Moderne}

Kürzlich testete Stiftung Warentest Antiviren- und Internetsicherheitssoftware. Die Gruppe "Anonymous" hörte vor einigen Wochen eine vertrauliche Telefonkonferenz zwischen dem FBI und Scotland Yard ab. Der Sicherheitsexperte Eugene Kaspersky warnte auf der diesjährigen Münchner Sicherheitskonferenz vor dem Cyberkrieg - das sind einige Meldungen, die zeigen, dass das Internet, das Nervensystem unserer Moderne, nicht sicher ist. In ihrem Beitrag „Höhere IT-Sicherheit durch Context Computer - Plädoyer für einen Paradigmenwechsel" haben Heinz-Jürgen Burkhardt und Rainer Prinoth das IT-Thema Internet-Sicherheit aufgegriffen. Will man die Ursachen beseitigen oder mindern, benötigt man einen Paradigmenwechsel, den sie als auf Arbeitskontexte hin konfigurierbare Context Computer beschrieben haben. Arbeitskontexte definieren dabei Aufgaben, Akteure und Sicherheitserfordernisse. Context Computer realisieren dann die benötigten, sicherheitstechnisch adäquat gekapselten Ausführungsumgebungen.

Django ist ein Python MVC Web-Framework für Rapid Development im Stil von Ruby on Rails. Trotz hoher Entwicklungsgeschwindigkeit ist Sicherheit für Django neben agilem Automatismus eines der Hauptdesignziele. Bastian Ballmann untersucht in seinem Artikel "Inside Django", wie das Framework vor den meisten typischen Sicherheitslücken in Webanwendungen schützt und zeigt auf, wo Entwickler trotz allem aufpassen müssen.

In komplexen Installationen informationstechnischer Systeme kooperiert eine große Zahl von Teilsystemen miteinander, deren Implementierungsmodelle völlig unterschiedlich sind. Auch wenn der Datenaustausch auf Basis genormter Protokolle erfolgt, ist die wichtigste Information, die Semantik der übertragenen Daten, oft nur unzureichend oder gar nicht bekannt. Selbst bei bekannter Semantik bleibt das Problem der zahlreichen und nicht unproblematischen Übersetzungen der Datensemantik in die jeweilige Semantik der Teilsysteme. Udo Döbrich und Roland Heidel skizzieren in ihrem Beitrag "Datengetriebene Programmsysteme" einen Ausweg aus dem Schnittstellenchaos.

Der IT-Arbeitsmarkt hat vom wirtschaftlichen Aufschwung 2011 kräftig profitiert. Die Zahl der Beschäftigten stieg, die bereits geringe Arbeitslosigkeit ist weiter zurückgegangen und die Nachfrage nach Fachkräften deutlich gestiegen. Im Bereich der hochqualifizierten IT-Spezialisten mehren sich Anzeichen für zunehmende Schwierigkeiten von Unternehmen bei der Besetzung offener Stellen, schreiben Ralf Beckmann und Franziska Hohnheiser in ihrem Beitrag "Gute Chancen im Aufschwung - Der Arbeitsmarkt für IT-Fachleute und Elektroingenieure".

Viel Vergnügen bei der Lektüre wünscht Ihnen

\section{Hermann Engesser, Chefredakteur}

